\title{
Lower Bounding Models for Wireless Networks
}

\author{
Jinfeng Du \\ ACCESS center and School of EE \\ Royal Institute of Technology \\ Stockholm, Sweden \\ Email: jinfeng@kth.se
}

\author{
Muriel Médard \\ Research Lab of Electronics \\ Massachusetts Institute of Technology \\ Cambridge, MA \\ Email:medard@mit.edu
}

\author{
Ming Xiao and Mikael Skoglund \\ ACCESS center and School of EE \\ Royal Institute of Technology \\ Stockholm, Sweden \\ Email: \{mingx, skoglund $\} @$ kth.se
}

\begin{abstract}
Motivated by the framework of network equivalence theory [1], [2], we present capacity lower bounding models for wireless networks by construction of noiseless networks which can be used to calculate an inner bound for the corresponding wireless network. We first extend the "one-shot" lower bounding model [6] to many-user scenarios, and then propose a two-step update of the one-shot models to incorporate the broadcast nature of wireless transmission. The main advantage of the proposed lower bounding method is its simplicity and the fact that it can be easily extended to larger networks. We demonstrate by examples that the resulting lower bounds can even approach the capacity in some setups.
\end{abstract}

\section{INTRODUCTION}

In [1] a new theory of network equivalence established the equivalence of a point-to-point noisy channel and a noiseless point-to-point bit-pipe as long as the throughput of the latter equals the capacity of the former. For independent multi-terminal networks, such as the multiple-access channel (MAC), the broadcast channel (BC), and the interference channel (IC), with two sources and/or two destinations, both upper and lower bounding models were proposed in [2]-[4]. The bounding accuracy was investigated in [2] in terms of both multiplicative and additive gaps between the upper and lower bounds for general noisy networks containing a collection of independent channels. In [3] the additive gap for Gaussian networks and the multiplicative gap for binary networks were specified for the cases when such noisy networks are only composed of point-to-point and two-user MAC/BC channels. The bounds obtained from network equivalence tools [2]-[4] can be tight in some setups [4, Lemma 3.2]. By applying the bounding models for the point-to-point channel [1] and the two-user BC [2], [4] to a frequency-division AWGN relay network, the capacity region was fully characterized in [5] in the wideband (i.e., low SNR) regime if the BC originating from the source node is physically degraded, or if the source treats the stochastically degraded BC as physically degraded. A class of one-shot bounding models were proposed in [6] for the general MAC and for two-user BC with independent noise, where channel emulation is done for each time instance. The approach is hence called "one-shot" in contrast to [1], [2] where channel emulation is done over infinite number of channel uses based on a lossy source coding argument for upper bounding models and a channel coding argument for lower bounding models. As illustrated in Fig. 1 for the $m$ user MAC and the two-user BC with independent noise, the
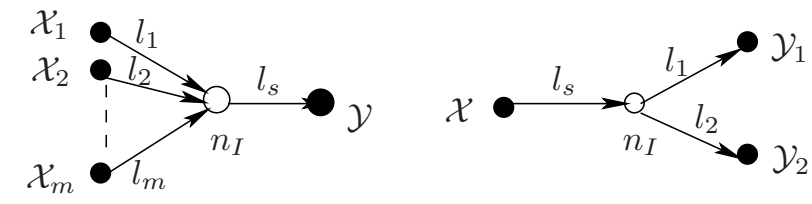

Fig. 1. The one-shot bounding models developed in [6] for $m$-user MAC and two-user BC. The white nodes indicated by $n_{I}$ are auxiliary operation nodes, and all the channels $l_{i}$ are noiseless point-to-point bit-pipes.

one-shot bounding model introduces auxiliary operation nodes $n_{I}$ to specify the rate constraints on the sum rate via bit-pipe $l_{s}$ and on each of the individual rates via bit-pipe $l_{i}$, and therefore can be represented by a rate vector $\left(R_{l_{s}}, R_{l_{1}}, R_{l_{2}}, \cdots\right)$. A layering approach with a global information flow routing technique was proposed in [7] for non-coupled wireless networks ${ }^{1}$, and this approach was proven to be approximately optimal in the sense that the resulting capacity lower bounds are within a multiplicative gap from the cut-set bound.

In this paper we provide a systematic scheme to construct capacity lower bounding models for general wireless networks, where the dependence between coupled BC and MAC components is handled by a two-step update procedure. As demonstrated by examples, the resulting inner bounds can approach capacity in some setups. Note that in this paper we focus on constructing noiseless bounding networks that can serve as the basis to compute capacity inner bounds, rather than finding the capacity of a noiseless network which itself is a very difficult problem [8], [9]. Our results on upper bounding models can be found in [10].

The rest of the paper is organized as follows. We first extend the one-shot lower bounding model to the $m$-user MAC and $\mathrm{BC}$ in Sec. II and then use them to construct lower bounding models for non-coupled networks in Sec. III. In Sec. IV we present the two-step update method for coupled networks and conclude this paper in Sec. V.

\section{II. $m$-USER ONE-SHOT LOWER BOUNDING MOdels}

The lower bounding model for the 2-user MAC in [6] can be extended straightforwardly to the $m$-user MAC by choosing an operating point in the capacity region of the MAC assuming independent sources. We choose the point that can be achieved

\footnotetext{
${ }^{1} \mathrm{~A}$ network is said to be coupled if any of its point-to-point connections is part of a MAC and a BC simultaneously. Otherwise it is non-coupled.
} 
TABLE I

RATE CONSTRAINTS IN THE LOWER BOUNDING MODEL FOR NON-DEGRADED BROADCAST CHANNELS WITH $m$ RECEIVERS.

\begin{tabular}{|c|c|c|c|c|c|}
\hline Rate $R_{i}, i=$ & 1 & 2 & 3 & $\cdots$ & $2^{m}-1$ \\
\hline$i:\{0,1\}^{m}$ & $0 . .001$ & $0 . .010$ & $0 . .011$ & $\cdots$ & $1 . .111$ \\
\hline$\left\{\mathcal{D}_{n}\right\}, n=$ & 1 & 2 & 2,1 & $\cdots$ & $m, \ldots, 3,2,1$ \\
\hline
\end{tabular}

by successive decoding, from the strongest received signal to the weakest.

The lower bounding model for the 2-user BC in [6] only considers private messages to each receiver. For the $m$-user $\mathrm{BC}$, each of these private messages dedicated for one specific receiver may also be decoded by another receiver due to the broadcast nature of wireless transmission, and such overheard messages can be useful when this $m$-user BC is part of a larger network. We therefore introduce a rate vector of length $2^{m}$

$$
\boldsymbol{R}=\left[R_{i}: i=0,1, \ldots, 2^{m}-1\right],
$$

to represent the lower bounding model for the $m$-user $\mathrm{BC}$. As illustrated in Table $\mathrm{I}, R_{i}$ is the rate constraint to ensure simultaneously successful decoding of the multicast (or unicast if $\left.i=2^{n-1}, n=1, \ldots, m\right)$ message by a subset of receivers. The intended receivers are given by the locations of ' 1 ' in the length- $m$ binary expression of the index $i$. For example, $R_{3}$ is the constraint for the multicast rate to receivers $\mathcal{D}_{2}$ and $\mathcal{D}_{1}$, and $R_{2^{m}-1}$ is the constraint for multicast rate to all receivers. Note that (1) contains both point-to-point bit-pipes (carrying unicast messages) and point-to-points bit-pipes (i.e., hyper-arcs carrying multicast messages). The constraint on the sum rate, represented by $R_{0}$, is defined as the sum of all the individual rate constraints, i.e., $R_{0} \triangleq \sum_{i=1}^{2^{m}-1} R_{i}$.

For non-degraded channels, the rate vector $\boldsymbol{R}$ can contain at most $2^{m}$ non-zero entries in the worst case scenario. However, for statistically degraded $m$-user BCs (e.g., scalar Gaussian $\mathrm{BCs}$ ), the number of non-zero entries in $\boldsymbol{R}$ can be limited to at most $m+1$ by creating a physically degraded channel via proper coding schemes ${ }^{2}$. We illustrate this by a $m$-user Gaussian BC.

\section{A. Example: Gaussian BC with $m$ Receivers}

Consider a Gaussian BC $\left(\mathcal{X}, p(\boldsymbol{y} \mid x), \prod_{i=1}^{m} \mathcal{Y}_{i}\right)$ where $Y_{i}$ is the output signal at receiver $\mathcal{D}_{i}$ with received $\operatorname{SNR} \gamma_{i}$ (the noise power is assumed to be 1 throughout this paper). Without loss of generality, assuming $\gamma_{1} \leq \gamma_{2} \leq \cdots \leq \gamma_{m}$, we divide the total information into $m$ distinct messages $\left\{W_{i}, i=1, \ldots, m\right\}$. By superposition coding of $W_{i}$ with power allocation parameter $\beta_{i} \in[0,1], \sum_{i=1}^{m} \beta_{i}=1$ at the source and successive interference cancellation at each receiver, successful decoding of $W_{i}$ can be realized at a set of receivers $\left\{\mathcal{D}_{n}, n=i, \ldots, m\right\}$ at multicast/unicast rate

$$
R_{2^{m}-2^{i-1}}<\mathcal{C}\left(\frac{\beta_{i} \gamma_{i}}{1+\gamma_{i} \sum_{j=i+1}^{m} \beta_{j}}\right)
$$

\footnotetext{
${ }^{2}$ Although such coding strategy is not optimal in general for stochastically degraded BCs, the rate loss will vanish in low SNR regime [5] if the source treats the channel as if it were physically degraded.
}

where $\mathcal{C}(x) \triangleq \frac{1}{2} \log (1+x)$. For example, successful decoding of $W_{1}$ can be realized at all receivers with a multicast rate of $R_{2^{m}-1}$, and successful decoding of $W_{m}$ can only be realized at receiver $\mathcal{D}_{m}$ with a unicast rate of $R_{2^{m-1}}$. The resulting rate vector is therefore

$$
\boldsymbol{R}=\left[R_{0}, R_{2^{m}-2^{i-1}}: i=1, \ldots, m\right],
$$

where the sum rate constraint $R_{0}$ is

$$
\begin{aligned}
R_{0} & =\sum_{i=1}^{m} R_{2^{m}-2^{i-1}}=\sum_{i=1}^{m} \frac{1}{2} \log \left(\frac{1+\gamma_{i} \sum_{j=i}^{m} \beta_{j}}{1+\gamma_{i} \sum_{j=i+1}^{m} \beta_{j}}\right) \\
& =\frac{1}{2} \log \left(1+\gamma_{1}\right)+\frac{1}{2} \sum_{i=2}^{m} \log \left(\frac{1+\gamma_{i} \sum_{j=i}^{m} \beta_{j}}{1+\gamma_{i-1} \sum_{j=i}^{m} \beta_{j}}\right) .
\end{aligned}
$$

The last equality comes from the fact that $\sum_{i=1}^{m} \beta_{i}=1$. Since $\gamma_{i-1} \leq \gamma_{i}$, the function $f(x) \triangleq \frac{1+x \gamma_{i}}{1+x \gamma_{i-1}}$ is monotonically increasing on $x \in[0,1]$, with its maximum $\frac{1+\gamma_{i}}{1+\gamma_{i-1}}$ achieved when $x=1$. It is straightforward to show

$$
R_{0} \leq \mathcal{C}\left(\gamma_{m}\right)
$$

where the equality is achieved when $\beta_{m}=1$ (i.e, $\beta_{i}=0$ for all $i \neq m)$. On the other hand, the sum-rate is upper bounded by

$$
R_{B C}=\frac{1}{2} \log \left(1+\sum_{i=1}^{m} \gamma_{i}\right)
$$

which can be achieved only if full cooperation among all receivers is possible. The gap between the upper and the lower bounds on the sum rate, measured in bits per channel use, is

$$
\Delta_{B C}=\frac{1}{2} \log \left(\frac{1+\sum_{i=1}^{m} \gamma_{i}}{1+\gamma_{m}}\right)<\frac{1}{2} \log (m),
$$

where the inequality comes from the assumption $\gamma_{i} \leq \gamma_{m}$ for all $i$. Hence, for the $m$-user Gaussian BC with receivers in isolation, feedback and receiver cooperation can increase the sum capacity by at most $\frac{1}{2} \log (m)$ bits. The gap becomes considerably smaller at low SNR or when the SNR for each link diverges. For example, with $\gamma_{1}=1, \gamma_{2}=2, \gamma_{3}=100$ (e.g, 0 , $3,20 \mathrm{~dB}$, respectively), the gap

$$
\Delta_{B C}=\frac{1}{2} \log \left(\frac{1+1+2+100}{1+100}\right) \approx 0.02,
$$

is much smaller than $\frac{1}{2} \log (3) \approx 0.79$.

\section{Bounding Models FOR NON-COUPLED NeTwORKS}

We illustrate the bounding procedure by a non-coupled noisy network $\mathfrak{N}$ shown in Fig. 2, where the source node $\mathcal{S}_{1}$ multicasts the message $W_{1}$ at rate $R_{1}$ to the destinations $\mathcal{D}_{1}$ and $\mathcal{D}_{3}$, and $\mathcal{S}_{2}$ multicasts $W_{2}$ at rate $R_{2}$ to the destinations $\mathcal{D}_{2}$ and $\mathcal{D}_{3}$, with the aid of a full-duplex relay $\mathcal{R}$. The point-topoint connections $\mathcal{S}_{1}-\mathcal{D}_{1}$ and $\mathcal{S}_{2}-\mathcal{D}_{2}$ are independent binary symmetric channels with crossover probability $\epsilon_{1}$ and $\epsilon_{2}$, respectively. The MAC ending at $\mathcal{R}$ and the $\mathrm{BC}$ originating from $\mathcal{R}$ are Gaussian channels with effective link SNR $\gamma_{i r}$ and $\gamma_{r j}, i=1,2, j=1,2,3$, respectively, and the relative order will determine the structure of the lower bounding model. 

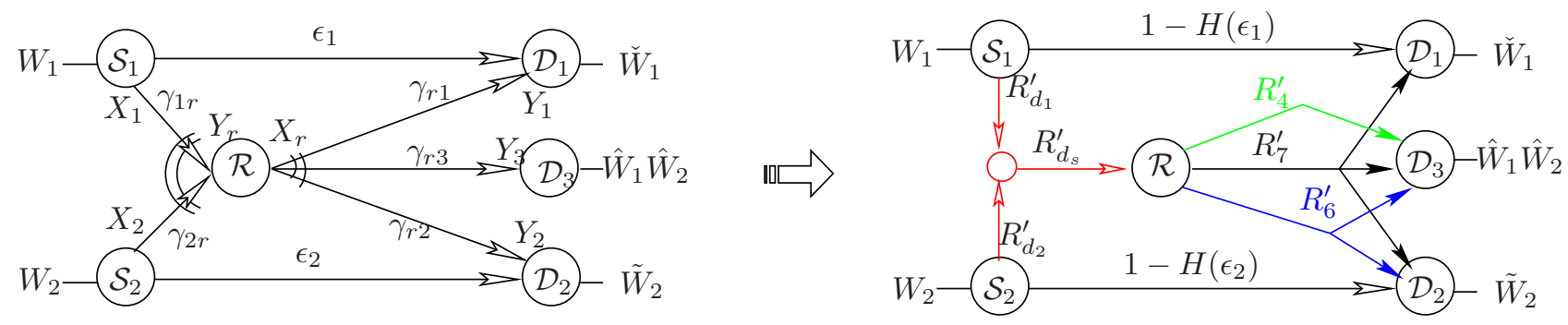

Fig. 2. A non-coupled noisy network $\mathfrak{N}$ (left) with independent binary symmetric channels, a 2-user MAC and a 3-user BC, and one example of its lower bounding networks (right) consisting of noiseless point-to-point and point-to-points bit-pipes (carrying multicast messages at rate $R_{6}^{\prime}$ and $R_{7}^{\prime}$, respectively).

By [1], the point-to-point connection $\mathcal{S}_{1}-\mathcal{D}_{1}$ (resp. $\mathcal{S}_{2}-\mathcal{D}_{2}$ ) can be replaced by a noiseless bit-pipe with throughput equal to its capacity $1-H\left(\epsilon_{1}\right)$ (resp. $1-H\left(\epsilon_{2}\right)$ ), without affecting the capacity of the original network. For the MAC with link SNRs $\gamma_{1 r}$ and $\gamma_{2 r}$, the lower bounding model is given by

$$
\mathfrak{C}_{l, M A C}=\left(R_{d_{s}}^{\prime}, R_{d_{1}}^{\prime}, R_{d_{2}}^{\prime}\right)
$$

where

$$
\begin{aligned}
R_{d_{s}}^{\prime} & =\mathcal{C}\left(\gamma_{1 r}+\gamma_{2 r}\right), \\
R_{d_{1}}^{\prime} & =\left\{\begin{array}{cc}
\mathcal{C}\left(\gamma_{1 r}\right), & \gamma_{1 r} \geq \gamma_{2 r}, \\
R_{d_{s}}^{\prime}-\mathcal{C}\left(\gamma_{2 r}\right), & \gamma_{1 r}<\gamma_{2 r},
\end{array}\right. \\
R_{d_{2}}^{\prime} & =R_{d_{s}}^{\prime}-R_{d_{1}}^{\prime} .
\end{aligned}
$$

The lower bounding model for the BC can be constructed according to (3) based on the realization of the channel SNRs $\gamma_{r j}, j=1,2,3$. Assuming we have $\gamma_{r 1} \leq \gamma_{r 2} \leq \gamma_{r 3}$, the corresponding lower bounding can be written as

$$
\mathfrak{C}_{l, B C}=\left[R_{0}^{\prime}, R_{4}^{\prime}, R_{6}^{\prime}, R_{7}^{\prime}\right]
$$

where

$$
\begin{aligned}
R_{7}^{\prime} & =\mathcal{C}\left(\frac{\gamma_{r 1} \beta_{1}}{1+\gamma_{r 1}\left(1-\beta_{1}\right)}\right), \\
R_{6}^{\prime} & =\mathcal{C}\left(\frac{\gamma_{r 2} \beta_{2}}{1+\gamma_{r 2}\left(1-\beta_{1}-\beta_{2}\right)}\right), \\
R_{4}^{\prime} & =\mathcal{C}\left(\gamma_{r 3}\left(1-\beta_{1}-\beta_{2}\right)\right), \\
R_{0}^{\prime} & =R_{4}^{\prime}+R_{6}^{\prime}+R_{7}^{\prime},
\end{aligned}
$$

with $\beta_{1}, \beta_{2} \geq 0, \beta_{1}+\beta_{2} \leq 1$ as power allocation parameters for superposition encoding at $\mathcal{R}$. As explained in Sec. II, $R_{7}^{\prime}$ represents the multicast rate to all the destination nodes, $R_{6}^{\prime}$ represents the multicast rate to destinations $\mathcal{D}_{2}$ and $\mathcal{D}_{3}$, and $R_{4}^{\prime}$ represents the unicast rate to $\mathcal{D}_{3}$. Note that the lower bounding model in (5) will be different when the order of channel SNRs changes. The resulting lower bounding network $\mathfrak{C}_{l}$ is shown in Fig. 2, where the redundant bit-pipe constraint $R_{0}^{\prime}$ is removed (therefore the auxiliary node for the $\mathrm{BC}$ is merged into $\mathcal{R}$ ).

Given each valid realization of $\left(\beta_{1}, \beta_{2}\right)$, there is a valid lower bounding network $\mathfrak{C}_{l}\left(\beta_{1}, \beta_{2}\right)$ for the original network $\mathfrak{N}$, and the corresponding achievable rate region can be described by the pentagon

$$
\boldsymbol{C}_{l}\left(\beta_{1}, \beta_{2}\right)=\left\{\begin{array}{l}
0 \leq R_{1} \leq \min \left\{1-H\left(\epsilon_{1}\right)+R_{7}^{\prime}, R_{d_{1}}^{\prime}\right\} \\
0 \leq R_{2} \leq \min \left\{1-H\left(\epsilon_{2}\right)+R_{6}^{\prime}, R_{d_{2}}^{\prime}\right\} \\
R_{1}+R_{2} \leq \min \left\{R_{4}^{\prime}+R_{6}^{\prime}+R_{7}^{\prime}, R_{d_{s}}^{\prime}\right\}
\end{array}\right.
$$

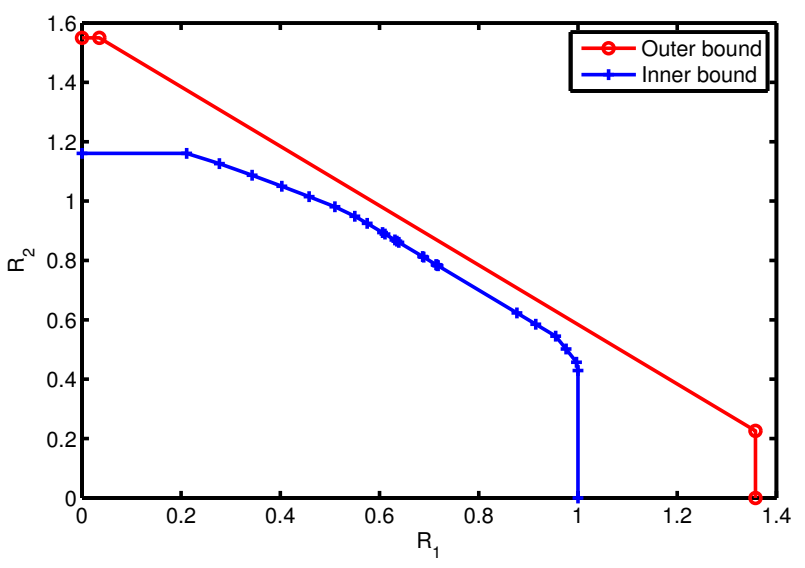

Fig. 3. The capacity inner bound for the noisy network $\mathfrak{N}$ obtained from its lower bounding network $\mathfrak{C}_{l}$, with $\epsilon_{1}=0.02, \epsilon_{2}=0.04, \gamma_{1 r}=3, \gamma_{2 r}=4$, $\gamma_{r 1}=1, \gamma_{r 2}=2$, and $\gamma_{r 3}=8$. The outer bound is obtained from its upper bounding network $\mathfrak{C}_{u}$ developed in [10].

We then take the union of all achievable rate regions to find the final capacity inner bound

$$
\boldsymbol{C}_{l}\left(R_{1}, R_{2}\right)=\underset{\beta_{1}, \beta_{2} \geq 0, \beta_{1}+\beta_{2} \leq 1}{\bigcup_{l}\left(\beta_{1}, \beta_{2}\right) .}
$$

As shown in Fig. 3, we have compared the capacity inner bound $C_{l}\left(R_{1}, R_{2}\right)$ obtained from the noiseless bounding networks $\mathfrak{C}_{l}$ and the outer bound developed in [10], for the original noisy network $\mathfrak{N}$ with $\epsilon_{1}=0.02, \epsilon_{2}=0.04, \gamma_{1 r}=3$, $\gamma_{2 r}=4, \gamma_{r 1}=1, \gamma_{r 2}=2$, and $\gamma_{r 3}=8$. The gap between the inner and the outer bound is within 0.1 bits on the sum rate, and within 0.4 bits on individual rates.

\section{Bounding Models for COUPLED Networks}

The lower bounding model designed for non-coupled networks assumes isolated source/destination nodes, which is not the case in wireless networks. A transmit signal can be designed for multiple destinations (as in BC) and the received signal may consist of signals from several source nodes (as in MAC) and thus interfere with each other. When a noisy connection between two nodes is part of both a BC and a MAC, the bounding models have to be updated. We demonstrate how the update is done step by step as follows. Note that the proposed method will ensure a valid lower bounding network, without claiming its optimality. 


\section{A. Inner Bounds with Updated Lower Bounding Models}

\section{Step I: Network Decomposition}

Given a noisy network, we first identify all the broadcast and multiple-access channels. For each $m$-user BC, we introduce an auxiliary node with one input channel connected to the transmitter, and $m$ output channels each connected to a receiver. For each $n$-user MAC, we introduce an auxiliary node with $n$ input channels each connected to a transmitter, and one output channel connected to the receiver. If a node is both the receiver of a MAC and the transmitter of a $\mathrm{BC}$, we introduce two auxiliary nodes, one for the receiving functionality and one for the transmitting functionality.

Step II: Apply Lower Bounding Models for point-topoint, BC, and Non-coupled MAC Channels

We replace each point-to-point channel with a bit-pipe of the same capacity. Each BC and each non-coupled MAC, i.e., a MAC where none of its input signals is part of the output of a $\mathrm{BC}$, is replaced with the corresponding lower bounding models as described in Sec. II.

Step III: Construct Lower Bounds for Coupled MAC

If (some of) the input signals to a MAC are the output signals from BCs, part of the received signals cannot be decoded and therefore behaves as interference. The original lower bounding models for non-coupled MAC, which assumes that all input signals can be decoded, need to be updated based on the sum power of the interfering signals. This can be calculated by taking into account the signal structure of each input source node. We illustrate this procedure by a coupled $m$-user Gaussian MAC.

\section{Example: Gaussian MAC with $m$ Transmitters}

Consider a Gaussian MAC $\mathfrak{N}=\left(\prod_{i=1}^{m} \mathcal{X}_{i}, p(y \mid \boldsymbol{x}), \mathcal{Y}\right)$, where $X_{i}$ is an input signal with $\mathrm{SNR} \gamma_{i}$. If $X_{i}$ can only be observed by the receiver in channel $\mathfrak{N}$, all the components of $X_{i}$ can be fully decoded by the receiver. If $X_{i}$ is the transmitted signal from a broadcasting source node, it may contain components that are not intended to be decoded by the receiver owing to rate and power allocation at the broadcast node, as described in Sec. II. The component of $X_{i}$ that cannot be decoded by the receiver behaves as interference during the decoding process. We denote the power of the interfering component by $\Gamma_{i}$, and the exact value can be obtained from the power allocation parameters chosen by the corresponding BC that transmits $X_{i}$. We have $\Gamma_{i}=0$ if all messages contained in $X_{i}$ are intended for successful decoding, and $\Gamma_{i}=\gamma_{i}$ if nothing is to be decoded. After careful examination of the structure of all the input signals, we can calculate the total power of interfering components contained in $Y$ as follows

$$
P_{I}=\sum_{i=1}^{m} \Gamma_{i}
$$

out of which

$$
P_{I, i}=P_{I}-\Gamma_{i}=\sum_{j \neq i} \Gamma_{j}
$$

is the amount of interference power introduced by input signals other than $X_{i}$. We call $P_{I, i}$ the "extrinsic interference" of $X_{i}$.
We can now construct the lower bounding model for the MAC $\mathfrak{N}$ based on the effective SNR, which is defined as

$$
\hat{\gamma}_{i}=\frac{\gamma_{i}-\Gamma_{i}}{1+P_{I}}, i=1, \ldots, m
$$

\section{Step IV: Rate Adjustment for Coupled BCs}

For $i=1, \ldots, m$, let a noisy connection $X-Y_{i}$ be part both of a $m$-user BC transmitting $X$ and a MAC whose received signal is $Y_{i}$, and denote the corresponding link SNR by $\gamma_{i}$. After Step III, we can obtain from (8) the extrinsic interference power $P_{I, i}$, caused by input signals other than $X$ in the coupled MAC with output $Y_{i}$. Without loss of generality, assuming $\gamma_{1} \leq \ldots \leq \gamma_{m}$, the rate constraints $R_{2^{m}{ }_{-2}{ }^{i-1}}^{\prime}$ defined in (2) should be adjusted by taking into account the extrinsic interference power $P_{I, k}$ for all $k \in S_{i} \triangleq\{i, \ldots, m\}$, i.e.,

$$
R_{2^{m}-2^{i-1}}^{\prime}=\min _{k \in S_{i}} \mathcal{C}\left(\frac{\gamma_{k} \beta_{i}}{1+P_{I, k}+\gamma_{k} \sum_{j=i+1}^{m} \beta_{j}}\right) .
$$

The sum rate constraint is adjusted accordingly. Note that the minimum operation in (10) comes from the fact that given $\gamma_{i} \leq \ldots \leq \gamma_{m}$ we cannot guarantee

$$
\frac{\gamma_{i}}{1+P_{I, i}} \leq \ldots \leq \frac{\gamma_{m}}{1+P_{I, m}} .
$$

Here we simply keep the structure of the original lower bounding model unchanged without claiming its optimality.

Step V: Construct an Inner Bound for the Resulting Noiseless Network

The resulting lower bounding network consists of only noiseless bit-pipes, but it may contain hyper-arcs (point-topoints bit-pipe) that carry the same data from one point to multiple points if the original noisy network has broadcast channels. The problem of finding the optimal scheme to manage the data flows over such noiseless networks is in general open. However, there exist many heuristic (and thus suboptimal in general) methods, see [11] for example, for constructing a valid inner bound.

\section{Step VI: Take the Union of all Valid Inner Bounds}

When superposition coding is used in constructing the lower bounding model for a $\mathrm{BC}$, there are an infinite ${ }^{3}$ number of lower bounding models due to the power allocation parameters. We can construct a valid lower bound for each of these bounding models and then take the union of all valid lower bounds to form the final inner bound.

\section{B. Illustrative Example: Multiple Multicast Relay Networks}

We illustrate the performance of our lower bounding methods based on a generic ${ }^{4}$ wireless multicast relay network shown in Fig. 4, where two source nodes $\mathcal{S}_{1}$ and $\mathcal{S}_{2}$, connected with a two-way backhaul (of rate $C_{12}$ and $C_{21}$ ), multicast the message $W_{1}$ at rate $R_{1}$ and $W_{2}$ at rate $R_{2}$, respectively, to both destinations $\mathcal{D}_{1}$ and $\mathcal{D}_{2}$ through Gaussian channels, with an aid from a full-duplex relay node $\mathcal{R}$.

\footnotetext{
${ }^{3}$ In practice we have a finite number of power allocation parameters, which may result in a slightly looser inner bound.

${ }^{4}$ By setting some parameters to zero, it covers many interesting networks.
} 


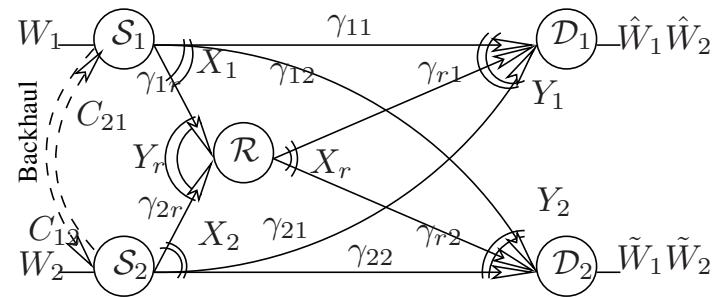

Fig. 4. A multiple multicast relay network with coupled MACs and BCs.

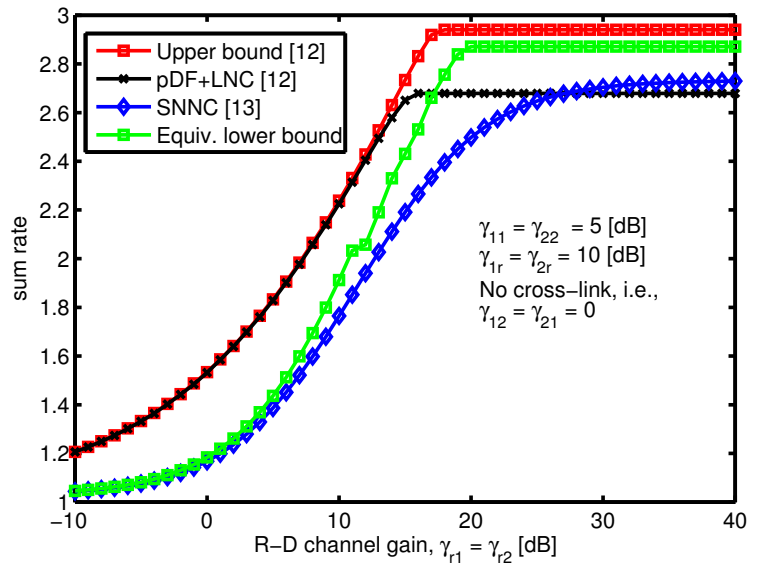

Fig. 5. Capacity bounds on sum rate for high-rate backhaul scenario without cross-links (i.e., $\gamma_{12}=\gamma_{21}=0$ ).

In Fig. 5 we focus on the high-rate backhaul scenarios and investigate the sum rate as a function of the relay-destination channel quality without cross-links (i.e., $\gamma_{12}=\gamma_{21}=0$ ). Our lower bounding methods discard any possibility of source cooperation and therefore suffers some performance degradation (less than 0.4 bits from the capacity upper bound [12]) in low and medium SNR regions. In the high SNR region where the network is limited by channel quality rather than source cooperation, our equivalence lower bound can approach the capacity (within 0.1 bits). The achievable rates obtained by the partial-decode-and-forward ( $\mathrm{pDF}+\mathrm{LNC}$ ) scheme [12] and by the short-message noisy network coding (SNNC) with message exchange scheme [13] are plotted as references.

In a low-rate backhaul scenario shown in Fig. 6, the capacity inner bound obtained from our lower bounding networks is "tight" in the sense that its gap from a genie-aided capacity outer bound developed in [12] is within 0.2 bits on individual rates and vanishes on sum rate. Interestingly, in this setup the equivalence inner bound outperforms the inner bounds obtained by pDF+LNC [12] and by SNNC [13].

\section{SUMMARY}

In this work we have presented capacity lower bounding models for wireless networks. We have extended the bounding models for the two-user BC to many-user scenarios and established the gap on the sum rate between the upper and lower bounding models. We have proposed a two-step update method to construct lower bounds for coupled networks. It has been shown in [5] that network equivalence tools can provide

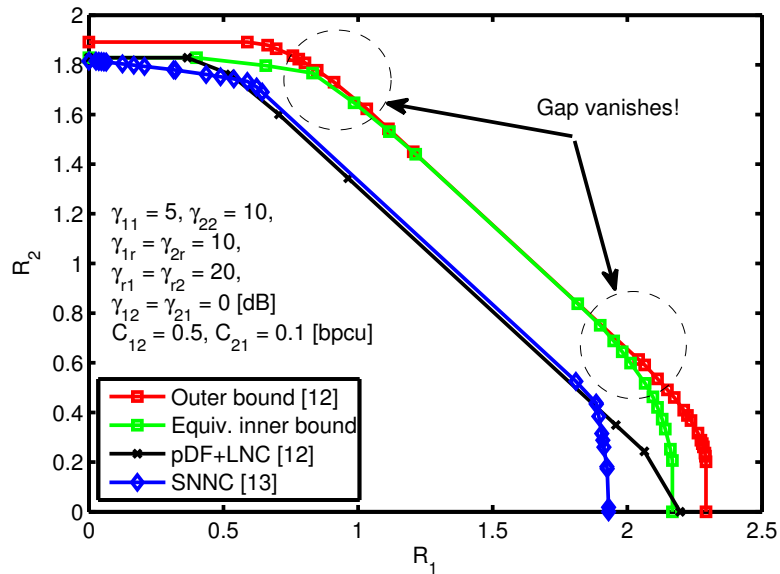

Fig. 6. Achievable rate regions in a low-rate backhaul scenario where the equivalence inner bound approaches the capacity region.

tight upper bound for some network setups. Interestingly, we have demonstrated by examples that network equivalence lower bounds can also approach capacity. The proposed lower bounding method, simple and computationally efficient, can be easily extended to larger networks. It therefore provides an additional powerful tool for characterizing the capacity region of general wireless networks.

\section{ACKNOWLEDGMENT}

This work is funded in part by the Swedish Research Council (VR). This material is based upon work supported by the Air Force Office of Scientific Research (AFOSR) under award No. FA9550-09-1-0196.

\section{REFERENCES}

[1] R. Koetter, M. Effros, and M. Médard, "A theory of network equivalence-part I: point-to-point channels," IEEE Trans. Inf. Theory, vol. 57, pp. 972-995, Feb. 2011.

[2] R. Koetter, M. Effros, and M. Médard, "A theory of network equivalence, part II," arXiv:1007.1033, Apr. 2010.

[3] M. Effros, "On capacity outer bounds for a simple family of wireless networks," in Proc. Inf. Theory \& App. Workshop, Feb. 2010.

[4] M. Effros, "Capacity Bounds for Networks of Broadcast Channels," in Proc. IEEE ISIT, Jun. 2010.

[5] N. Fawaz and M. Médard, "A Converse for the Wideband Relay Channel with Physically Degraded Broadcast," in Proc. IEEE Inf. Theory Workshop, Oct. 2011.

[6] F. P. Calmon, M. Médard, and M. Effros, "Equivalent models for multiterminal channels," in Proc. IEEE Inf. Theory Workshop, Oct. 2011.

[7] S. Kannan, A. Raja, and P. Viswanath, "Local phy + global flow: a layering principle for wireless networks," in Proc. IEEE ISIT, Aug. 2011.

[8] A. R. Lehman and E. Lehman, "Complexity classifications of network information flow problems," in Proc. 41st Allerton Conf., Sep. 2003.

[9] T. Chan and A. Grant, "Dualities between entropy functions and network codes," IEEE Trans. Inf. Theory, vol. 49, pp. 3129-3139, Oct. 2008.

[10] J. Du, M. Médard, M. Xiao, and M. Skoglund, "Capacity bounding models for wireless networks," manuscript, in preparation.

[11] D. Traskov, M. Heindlmaier, M. Médard, and R. Koetter, "Scheduling for network coded multicast," IEEE/ACM Trans. Networking, vol. 20, pp. 1479-1488, Oct. 2012.

[12] J. Du, M. Xiao, M. Skoglund, and M. Médard, "Wireless multicast relay networks with limited-rate source-conferencing," IEEE J. Sel. Areas Commun., accepted. DOI: 10.1109/JSAC.2013.130804.

[13] J. Du, M. Xiao, M. Skoglund, and S. Shamai (Shitz), "Short-message noisy network coding with partial source cooperation," in Proc. IEEE Inf. Theory Workshop, Sep. 2012. 